\title{
Testing a Quantum Error-Correcting Code on Various Platforms
}

Qihao Guo ${ }^{1,2,3, *}$, Yuan-Yuan Zhao ${ }^{4,3, *}$, Markus Grassl ${ }^{5,6}$, Xinfang Nie ${ }^{7,3}$, Guo-Yong Xiang ${ }^{4}$, Tao Xin $^{7,3}$, Zhang-Qi Yin ${ }^{8}, \&$ Bei Zeng ${ }^{9,10,11}$

${ }^{1}$ Institute for Quantum Computing, Baidu Research, Beijing 100193, China

${ }^{2}$ Department of Applied Physics, Xian Jiaotong University, Xian, Shaanxi 710049, China

${ }^{3}$ Center for Quantum Computing, Peng Cheng Laboratory, Shenzhen 518055, China

${ }^{4}$ CAS Key Laboratory of Quantum Information, University of Science and Technology of China, Hefei 230026, China

${ }^{5}$ Max Planck Institute for the Science of Light, 91058 Erlangen, Germany

${ }^{6}$ International Centre for Theory of Quantum Technologies, 80-308 Gdańsk, Poland

${ }^{7}$ Shenzhen Institute for Quantum Science and Engineering, Southern University of Science and Technology, Shenzhen 518055, China

${ }^{8}$ Center for Quantum Technology Research, School of Physics, Beijing Institute of Technology, Beijing 100081, China

${ }^{9}$ Department of Physics, The Hong Kong University of Science and Technology, Clear Water Bay, Kowloon, Hong Kong

${ }^{10}$ Department of Mathematics \& Statistics, University of Guelph, Guelph, Ontario, N1G 2W1, Canada

${ }^{11}$ Institute for Quantum Computing, University of Waterloo, Waterloo, Ontario, N2L 3G1, Canada

Quantum error correction plays an important role in fault-tolerant quantum information processing. It is usually difficult to experimentally realize quantum error correction, as it requires multiple qubits and quantum gates with high fidelity. Here we propose a simple quantum error-correcting code for the detected amplitude damping channel. The code requires only two qubits. We implement the encoding, the channel, and the recovery on an optical platform, the IBM Q System, and a nuclear magnetic resonance system. For all of these systems, the error correction advantage appears when the damping rate exceeds some threshold. We compare the features of these quantum information processing systems used and demonstrate the advantage of quantum error correction on current quantum computing platforms.

\section{Introduction}

Quantum computing, as the next generation of information technology, exploits the superposition principle and quantum entanglement to solve some difficult problems more efficiently than classical computing devices. It is widely believed that quantum computing has potential to realize an exponential advantage for certain problems, such as prime factor decomposition ${ }^{1}$ and principal component analysis $^{2}$, over current classical algorithms. In addition, some pioneering work also 
connects quantum computing with other research fields, including quantum simulation, cryptography, and machine learning. Since the concept of quantum computers came into being, several quantum systems, such as linear optical systems, nuclear magnetic resonance (NMR) systems, trapped ion systems, and superconducting circuits, were regarded as possible platforms to implement quantum computers ${ }^{3}$. Over the past decade, hardware for quantum computers has undergone an astonishing evolution, especially on superconducting circuits and trapped ion systems. Very recently, Google announced that they had achieved quantum advantage using a programmable superconducting processor with 53 qubits ${ }^{4}$. In the field of trapped ions, IonQ also made a presentation about their quantum computer with 79 processing qubits ${ }^{5}$. On the other hand, IBM and Rigetti released their online quantum platforms linking with real superconducting quantum computers to the public. We are now entering a new era in quantum technology, namely the Noisy Intermediate-Scale Quantum (NISQ) ${ }^{6}$ era, even with fault-tolerant quantum computing still a distant dream.

Theoretically, quantum computers could outperform classical computers dramatically. However, it still presents a major obstacle that the information encoded on qubits is very vulnerable to the noise induced by inevitable interaction between the qubits and the environment. Almost all the proposed physical implementations encounter quantum errors, including decoherence, imperfect quantum logic gates, and readout error. A direct approach to reduce quantum errors is improving the quantum computers on the physical level. At present, in superconducting quantum processors, single-qubit and two-qubit gate fidelities exceed $99.9 \%$ and $99.5 \%{ }^{4}$, respectively. Benefiting from well-developed quantum control techniques, such as composite pulses ${ }^{77}$, refocusing pulses ${ }^{8}$, and the Gradient Ascent Pulse Engineering (GRAPE) algorithm ${ }^{9}$, fidelities of quantum gates can reach even higher accuracy on NMR quantum computers.

While improving the quantum hardware is in the main focus of research right now, it is impossible to completely eliminate the errors in quantum computers. To realize a reliable quantum computer, additional techniques are required. Quantum error correction (QEC) ${ }^{[0 \mid 1112}$, protecting quantum information against unwanted operations, has spawned considerable interest from both physicists and mathematicians. Some initial theoretical results in this field focused on quantum error-correcting codes (QECC) $13|14| 15|16| 17$, other approaches are noiseless quantum codes and decoherence free subspaces ${ }^{18}$. The discovery of QECCs enhanced the possibility of building a quantum computer and has further lead to the concept of fault-tolerant quantum computation 1719220 . One important QECC is the surface code with a fault tolerance threshold of $1 \times 10^{-2}$ for each error source $^{21 \mid 22}$. Previous experimental progress for some quantum error-correcting codes demonstrated the power of QECC for several qubits for linear optics ${ }^{23}$, trapped ions ${ }^{2425}$, NMR ${ }^{26}$, and superconducting circuits $27 / 28 \mid 29130$. Measurement-based feedback ${ }^{30 \mid 31}$ and other advanced techniques have also been developed to implement error correction, in order to build a continuous-time and automatic quantum error correction system.

In this paper, we report on the implementation of a channel-adapted detected amplitude quantum code using a two-qubit system on various platforms: a quantum optical system, the IBM Q 


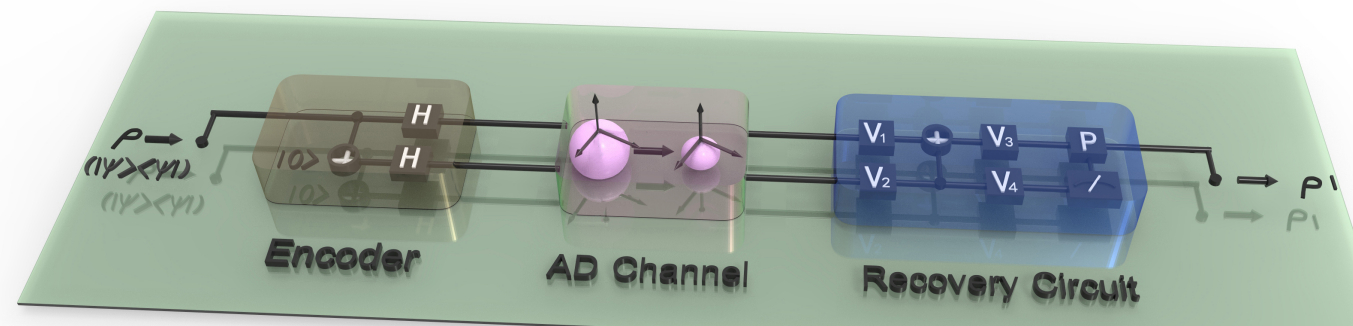

Figure 1: The model of the quantum communication system with an amplitude damping channel. The encoder maps an arbitrary initial single-qubit state $\rho=|\psi\rangle\langle\psi|$ to the code space using two qubits. Then a detected amplitude damping channel acts on each of the two qubits. Finally we apply the recovery circuit (including decoding), which discards the second qubit, obtaining a single-qubit output state $\rho^{\prime}$ that ideally has a large overlap with the input state $|\psi\rangle$.

Experience superconducting circuit, and an NMR quantum system. The experiments on different quantum systems successfully demonstrate the power of the error-correcting code with observable improvement of the fidelity when the damping rate is larger than a threshold $\gamma_{c}$.

\section{Result}

QECC for Detected Amplitude Channel In a typical quantum information process, like the one shown in Fig. 1, quantum information might be subject to spontaneous decay with detected photon emission, which is modelled by the dectected amplitude channel. Generally, a dectected amplitude damping channel is composed of an amplitude damping channel (denoted by $\Phi_{A D}$, see the Supplementary Material) and an ancilla system indicating whether damping has ocurred. The channel can be described by Kraus operators with an extra qubit,

$$
\Phi_{D J}(\rho)=\sum_{i}\left(A_{i} \rho A_{i}^{\dagger}\right) \otimes|i\rangle\left\langle\left. i\right|_{\text {anc }},\right.
$$

where $A_{0}=\left(\begin{array}{cc}1 & 0 \\ 0 & \sqrt{1-\gamma}\end{array}\right)$ and $A_{1}=\left(\begin{array}{cc}0 & \sqrt{\gamma} \\ 0 & 0\end{array}\right)$.

The construction of quantum error-correcting codes for the detected amplitude channel has been discussed in Refs. 32/33/34, The simplest code correcting a single error of the detected amplitude channel needs only two qubits, and hence can be implemented on a present quantum computer. Based on the analysis in Ref. 32, we firstly encode the initial state $|\psi\rangle=\alpha|0\rangle+\beta|1\rangle$ onto the basis $|+\rangle|+\rangle$ and $|-\rangle|-\rangle$ using a CNOT gate followed by two Hadamard gates,

$$
\alpha|0\rangle+\beta|1\rangle \rightarrow \alpha|+\rangle|+\rangle+\beta|-\rangle|-\rangle .
$$


For the two-qubit code given by Eq. 2, there are two standard error correction protocols derived from the classical code, denoted by Standard A and Standard B. Additionally, using the polar decomposition method in the Supplementary Material, we obtain Optimal Recovery which will lead a higher fidelity. The optimal recovery operation will depend on the damping parameter $\gamma$, meaning it is a "channel-adaptive" error correction scheme. The detailed process for the different protocols is given in the Methods section.

Physical Systems Photons as a kind of "flying qubits" are widely used for quantum information processing and simulation. In a linear optics system, single qubit operations can be implemented with high fidelity as photons are essentially decoherence-free and are not affected by the environment. However, two-qubit gates, like the controlled-NOT (CNOT) gate, become a challenge as it is difficult to let photons interact. We are using optical qubits encoded in the polarization degree of freedom to demonstrate quantum error correction.

In 2018, IBM Q released a 14-qubit transmon superconducting quantum processor (Fig. 2](b)), IBM Q 16 Melbourne, which is accessible via Qiskit, an open-source framework for quantum computing on IBM Q Experience. The average fidelity of single qubit operations exceeds $99.0 \%$, and the fidelity of the CNOT operation is nearly $82.7 \%$ to $95.2 \%$. The pulse durations are $100 \mathrm{~ns}$ and $348 \mathrm{~ns}$ for single qubit rotation gates and CNOT gates based on the cross-resonance interaction, respectively. In addition, two-qubit gates are only permitted between neighboring qubits that are connected by a superconducting bus resonator (see the inset in Fig. 2(b)). More information on the qubits and quantum gates on IBM Q 16 Melbourne, such as the dephasing times and gate fidelities, can be found on the IBM Q site https: / / quantumexperience.ng.bluemix.net/qx/ devices.

Nuclear magnetic resonance (NMR) quantum computing is one of the first proposed schemes for building a quantum computer with spin- $1 / 2$ nuclei, such as ${ }^{1} \mathrm{H}$ or ${ }^{13} \mathrm{C}$. With a time-varying radio frequency (RF) field and the free evolution between the different spins, arbitrary unitary transforms can be implemented in the NMR quantum computer. In our experiment, we used a Crotonic acid specimen. The four qubits on the Crotonic acid are represented by the spin- $1 / 2{ }^{13} \mathrm{C}$ nuclear spins, labeled as $\mathrm{C}_{1}$ to $\mathrm{C}_{4}$ as shown in Fig. 2(c). The decoherence times of the Crotonic acid are $T_{1} \approx 1500 \mathrm{~ms}$ and $T_{2}^{*} \approx 550 \mathrm{~ms}$. All NMR experiments were carried out on a Bruker DRX $600 \mathrm{MHZ}$ spectrometer at room temperature.

Experimental Schemes For the three quantum systems, quantum optical platform, IBM Q superconducting circuit and NMR system (see the Supplementary Material), we have implemented different variants of quantum error-correction for the detected amplitude damping channel. In this model of decoherence, an excited state decays to the ground state with some probability. Monitoring the system, one obtains the addtional classical information whether the system has decayed or not. Owing to the features of the different systems, we first adapt our scheme to the particular device and decompose the quantum circuits into basic gates native for each system. In Fig. 3, we 

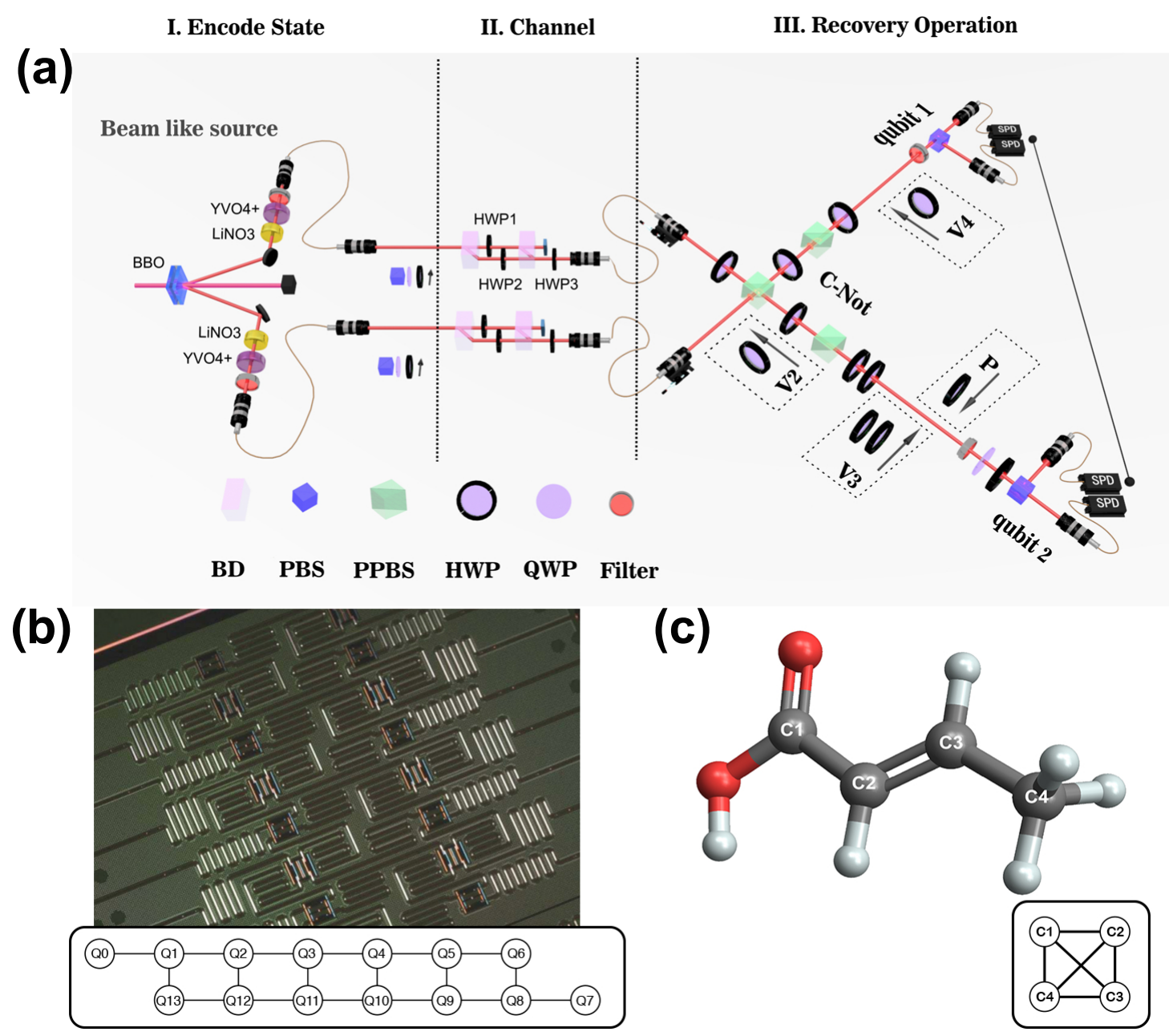

Figure 2: Illustration of the different quantum systems. (a) On the optical platform, we utilize a $390 \mathrm{~nm}$ femto-second light to pump a sandwich beamlike phase-matching $\beta$-barium-borate (BBO) crystal to generate pairs of polarization entangled photon as qubits. (b) IBM Q 16 Melbourne, consisting of 14 superconducting qubits connected via microwave resonators, together with the coupling structure. (c) The four qubits on the Crotonic acid are given by the spin- $1 / 2$ nuclear spins of ${ }^{13} C$. Each of the four spins couples to the other three.

give the quantum circuits that we employed in the realistic experimental process.

As shown in Fig. 2 (a), a $390 \mathrm{~nm}$ femto-second light (frequency-doubled from a $780 \mathrm{~nm}$ mode-lock Ti:sapphire pulsed laser with a pulse width of $150 \mathrm{fs}$ and repetition rate $76 \mathrm{MHz}$ ) pumps a sandwich beamlike phase-matching $\beta$-barium-borate (BBO) crystal to generate pairs of polarization entangled photon $\frac{1}{\sqrt{2}}(|H V\rangle+|V H\rangle)$ in the spontaneous parametric down-conversion (SPDC) process. Based on the entangled photons source, we can prepare the desired encoded state for the six different states (see the Supplementary Material) by using polarization beam splitters (PBS), 
half wave plates (HWP), and quarter wave plates (QWP). The detailed configurations are given in Table 1 in the Supplementary Materials.

As illustrated in the middle part of Fig. 2(a), for the optical platform we use an interferometer to implement the detected amplitude damping channel ${ }^{35}$. After passing through the first beam displacer (BD), the photons with horizontal polarization $(H)$ and vertical polarization $(V)$ are parallelly displaced with respect to each other ${ }^{36 / 37}$. For the operator $A_{0}$, the amount of damping $\gamma$ is adjusted by rotating HWP1 placed between two BDs by the angle $\theta$, with $\sin ^{2} 2 \theta=1-$ $\gamma$. Meanwhile HWP2 and HWP3 are rotated by $45^{\circ}$ to perform the bit-flip operator. Regarding $A_{1}$, HWP1 is rotated by $\theta$, where $\gamma=\sin ^{2} 2 \theta$. Both HWP2 and HWP3 are set at $0^{\circ}$ to remove the horizontally polarized photon (part II in Fig. 2(a)). Hence, the two interferometers together (middle part of Fig. 2 (a)) can simulate the four error pattern: $A_{0} A_{0}, A_{0} A_{1}, A_{1} A_{0}$, and $A_{1} A_{1}$. In the error correction part, we use the method of Refs. 38.39.40 to implement an all-optical CNOT gate, which is constructed by partially polarizing beam splitters (PPBS) and HWPs. To quantify the quality of the CNOT gate, we perform quantum process tomography showing that the fidelity between the implemented and the ideal gate is about $88.5 \%$. The errors are mainly caused by the mode mismatch of the Hong-Ou-Mandel (HOM) interferometer. In our experiment, the error patterns and the corresponding recovery operations are given in Table 2 , where the gates $H$ and $X$ can be easily realized by rotating the HWP by $22.5^{\circ}$ and $45^{\circ}$ respectively. The detailed information about the case without error correction is given in the Supplementary Materials.

On IBM Q and the NMR system we use two ancilla qubits to implement the two-qubit detected amplitude channel. The qubits of IBM Q 16 Melbourne and the Crotonic acid (see Fig. 22(b) and (c), resp.) meet the required coupling structure (other quantum chips from IBM Q do not match this connectivity map). To be more concrete, $Q_{5}, Q_{6}, Q_{8}, Q_{9}$ on IBM Q 16 Melbourne are selected because the average error rates of CNOTs between those qubits are lower than others. Generally, there are three parts in the quantum circuit, encoder, amplitude channel, and recovery circuit (containing the decoder) in the IBM Q and the NMR experiments, as shown in Fig. 3 (b). First, we prepare the initial state $|\psi\rangle$ by a single qubit rotation of $Q_{5}$. A CNOT gate and two Hadamard gates compose the encoder. With controlled-y-rotation gates $R_{y}(\theta)$ acting on the ancillas with the encoded qubits as control and CNOT gates acting on the encoded qubits, we can simulate the two-qubit detected amplitude channel ${ }^{[12}$. The relation between the damping ratio $\gamma$ and the rotation angle $\theta$ is $\gamma=\sin ^{2}(\theta / 2)$. Measuring the ancilla qubits reveals which type of error occurred. If the result is $|0\rangle, A_{0}$ has occurred on the corrsponding encoded qubit, while $A_{1}$ has occurred when the result is $|1\rangle$. Recovery circuits optimized for IBM $Q 16$ Melbourne and the NMR system are shown in Fig. 3(b). To extract the quantum density matrix of the decoded qubit, we use quantum state tomography (QST) and post selection (see the Supplemental Material), measuring the output of the same quantum circuit in different bases. For the IBM Q experiments, we construct the circuit with three-parameter single qubit rotation gates $U_{3}(\theta, \lambda, \phi)$ and CNOT gates. For the NMR experiments, we generate the pulse sequences of the encoder, two-qubit amplitude damping channel, and recovery circuit using an optimized shape pulse sequence with a total time of $61 \mathrm{~ms}$. 
(a)

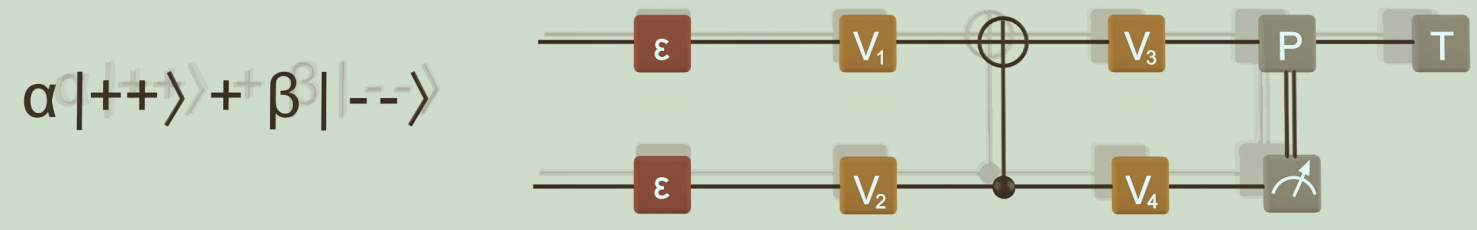

(b)

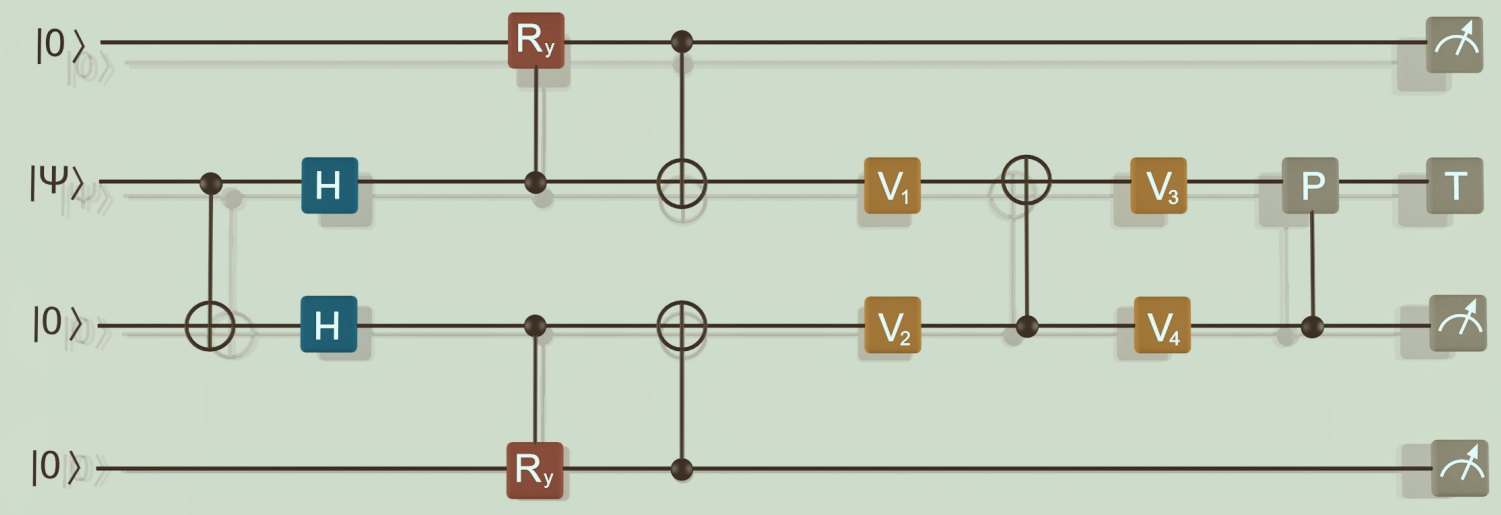

Figure 3: Quantum circuits for our experiments. (a) The optical platform. After generating pairs of entangled photon, we prepare the desired encoded state $\alpha|++\rangle+\beta|--\rangle$ with polarization beam splitters and wave plates, see Table 2. The detected amplitude damping channel is depicted by $\varepsilon$. In the error correction part, we implement Standard Correction A (see Supplemental Material) using four single qubit rotations and a CNOT based on a HOM interferometer. For the reconstruction of the decoded state, we use post-selection on the other qubits. (b) The circuit for IBM Q and the NMR system. Artificial amplitude damping channels are implemented by a controlled- $y$-rotation from the encoded qubits to the ancillas and the subsequent CNOT gates. Measuring the ancilla qubits at the end reveals which error has occurred. The single-qubit gates $V_{1}, V_{2}, V_{3}$, and $V_{4}$ in the recovery circuit depend on the particular error. To simplify the circuit, we run experiments with all settings and use post-selection on the corresponding measurement results of the ancillas. At the end, we use single-qubit state tomography on the second qubit to reconstruct the density matrix. 
Experimental Results The main experimental results for the three systems are shown in Fig. 4. The fidelity of the effective communication channel is plotted as a function of the damping parameter $\gamma$. For the three different systems, we show the effective regions for which Optimal Recovery (respectively Standard Correction A for the optical platform) yields a higher fidelity than using no error correction. Without error correction, the optical platform shows a great advantage in comparison to the other two systems, with the performance of IBM Q being the lowest. However, with error correction, the situation changes dramatically. For the optical platform, the state fidelity drops already a lot at $\gamma=0$, while adding error-correcting only slightly reduces the fidelity at $\gamma=0$ for IBM Q. Exhibiting the largest effective region (lighter blue), our error correction scheme exhibits a good performance on the NMR system, and the maximal improvement at $\gamma \approx 0.6$ reaches approximately 0.2 . For IBM Q, the improvement (red region) is smaller, but it is still given for a large range of damping parameters $\gamma$. For the optical platform, error correction improves the overall fidelity only a little for $\gamma>0.83$

On the optical platform (see Fig. S2 in the Supplementary Material) we perform experiments with Standard Correction A and without correction. At the mercy of the bad fidelity of implementing the CNOT by HOM interference, we find that at lower damping probabilities $(\gamma=0.17 \sim 0.83)$, the fidelity for the state without correction is larger than with standard correction. However, if the damping probability $\gamma$ is larger than 0.83 , standard error correction will be better. This demonstrates some limited improvement using quantum error correction.

Fig. S3 in the Supplementary Material shows the result for IBM Q averaging 4096 runs for 16 sample points. For $\gamma \in[0.0,0.36)$, no correction yields a higher fidelity than Optimal Recovery since "Without Correction" involves only two qubits. Generally, it is ubiquitous to QEC that the encoded states get worse initially as the encoding operations reduce the fidelity. The blue star plotted at $\gamma=0.36$ in Fig. S3 (a) indicates the intersection when the overall fidelity of "Optimal Recovery" equals "Without Correction". When the damping parameter $\gamma$ increases, both Standard Correction A and Standard Correction B show the capacity of error correction, but neither outperforms Optimal Recovery.

The results for the NMR system are show in Fig. S4. Optimal Recovery, Standard Correction A, as well as Standard Correction B show substantial improvements in comparison to Without Correction, indicating the power of quantum error correction. Furthermore, the state fidelity curves for Standard Correction A and Standard Correction B exhibit faster decay than the curve for Optimal Recovery, revealing that Optimal Recovery is indeed the best error correction scheme for the detected amplitude damping channel, which matches the theoretical result.

\section{Discussion}

The experiments mainly demonstrate the potential to realize quantum error correction on a quantum computer in the NISQ era by implementing an optimal error-correcting code for detected 


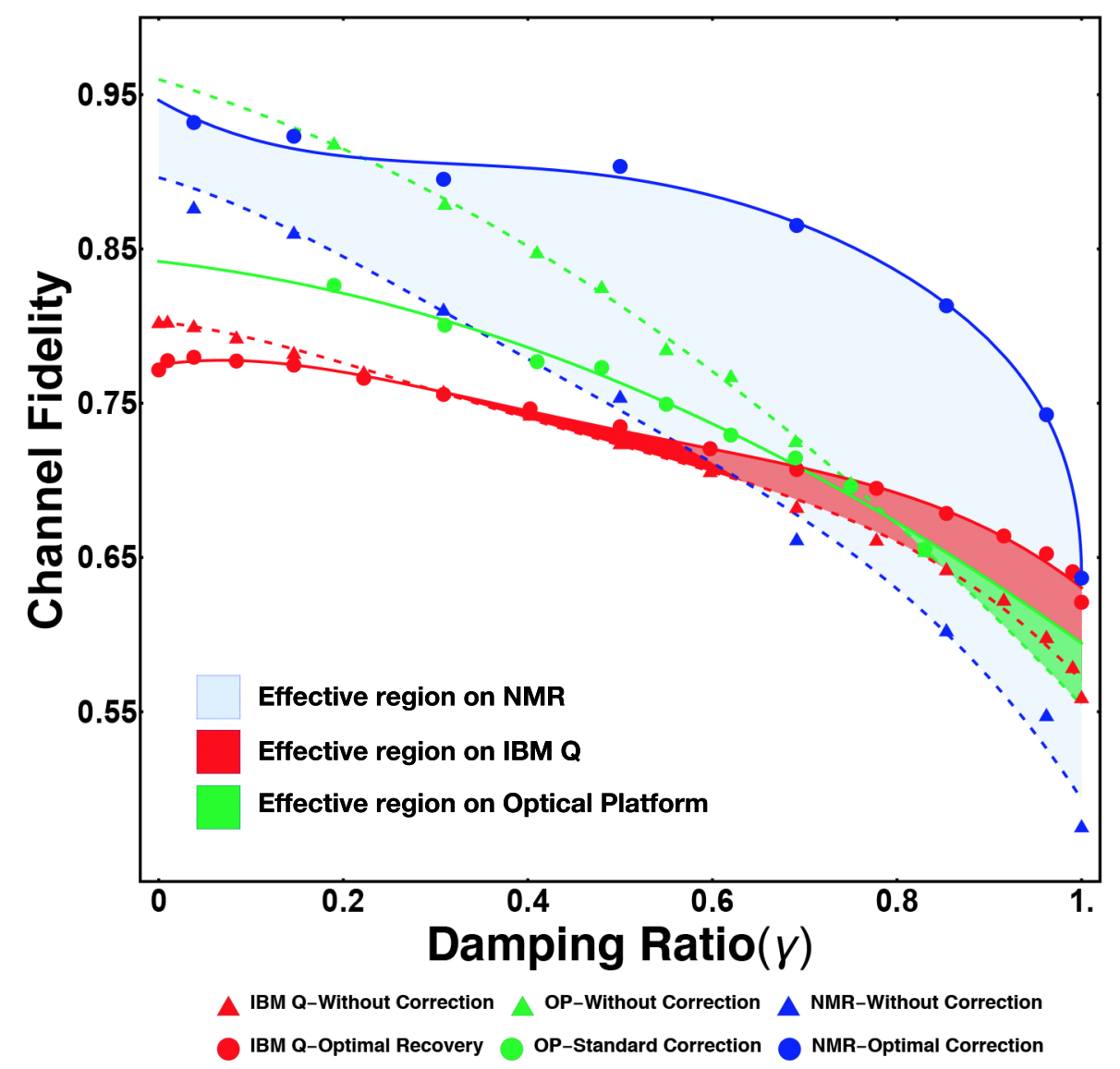

Figure 4: Comparison of the error correction capacity on the different systems. The red, green, and blue regions charactize the effective region enclosed by the fidelity curves for IBM Q, the optical platform (OP), and the NMR system, respectively. The solid and dashed lines are polynomial fits to our experiment data. The solid lines represent Optimal Recovery, and the dashed lines correspond to the case Without Correction. For IBM Q and the optical platform, when the damping ratio $\gamma$ is small, Optimal Recovery (resp. Standard Correction A) performs worse initially because of the limited fidelity of the additional encoding operations. When the damping ratio $\gamma$ increases, Optimal Recovery reveals its capacity gradually. 
amplitude damping on IBM Q, an optical platform, and an NMR system. All experiments provide evidence that the advantage of quantum error correction can even be revealed on a present quantum computer, with only a few qubits and faulty quantum gates. For all three systems, Optimal Recovery shows eventually an improvement in comparison to Without Correction. On the other hand, for small damping parameters $\gamma$, the correction scheme does not come into effect on the optical platform and on IBM Q.

Our experiments also reveal the underlying relation between the ability of quantum control and the performance of quantum error correction. In a typical quantum information process, quantum errors mainly stem from imprecise readout, decoherence, and faulty CNOT gates.

Firstly, we consider the influence of imprecise readout. The readout error for the optical platform and the NMR system can be neglected because for both the precision of readout is close to $99.9 \%$. On the IBM Q platform, however, the average error rate of readout is nearly $5.0 \%$, see Table 3 in the Supplementary Material. Apparently, the readout error only contributes a fraction of the entire infidelity in our experiments.

The qubit quality, especially the coherence time, is also an important factor for the performance of the quantum error-correcting code. A rough estimate for the state $\frac{1}{\sqrt{2}}(|0\rangle+|1\rangle)$ shows that the decoherence error contribution from $T_{2}$ for IBM Q and the NMR system are $3.5 \%$ and $9.9 \%$, respectively (see the Supplementary Material). Therefore, the decoherence is not the main source of the infidelity in IBM Q experiments, but may cause the dominant error in the NMR system.

For the optical system, substantial infidelity is contributed by the CNOT based on HOM interference. When adding the CNOT to the recovery circuit, the total shot numbers of photons will be suppressed by the PPBS crystal. We denote the phenomenon by shot loss. If we use ideal probabilities that the errors happen instead of the real probabilties in the experiments to reconstruct the effective density matrix, the correcting effect will enhance, see Fig. S5. A similar effect occurs for IBM Q because of cross-resonance CNOT gates. This phenomenon stems from $Z Z$-crosstalk in the superconducting qubit chips ${ }^{42}$. To reconstruct the density matrix with ideal probabilties, even Standard Correction A shows the capcity to improve the channel fidelity in Fig. S5. However, in the NMR experiments, we use the GRAPE algorithm to generate the total pulse sequence with a precision of $99.9 \%$, which gives a great improvement to CNOTs and other operations.

In conclusion, our experiments demonstrate that the quality of CNOT mainly influences the performance of quantum error correction. CNOT operations, at the core of both encoder and decoder, play a unique role to generate entanglement in both quantum error correction and quantum computing. Our results motivate further investigations to improve the precision of CNOT operations and indicate the route towards viable quantum error correction in the NISQ era. 


\section{Methods}

Standard Correction A/B For the two-qubit code given by Eq. (2), Standard Correction A/B protocols can be derived as follows:

- If $A_{0} A_{1}$ (or $A_{1} A_{0}$ ) happens, discard the qubit on which $A_{1}$ happened. On the other qubit, apply $X$ to compensate for the phase error introduced by $A_{1}$ acting differently on $|+\rangle$ and $|-\rangle$.

- If $A_{0} A_{0}$ happens, directly decode the two qubits.

- If $A_{1} A_{1}$ happens, the quantum state $|\psi\rangle$ is converted to the state $|00\rangle$. To maximize the fidelity, we transform it to an equally weighted superposition state $\frac{1}{\sqrt{2}}(|0\rangle+|1\rangle)$. There are two different schemes to create an equally weighted superposition state which we refer to as Standard Correction A/B (see Table. 2), respectively.

Optimal Recovery The Optimal Recovery operators are derived in the Supplementary Material. We find a pair of recovery operations $V_{3}$ and $V_{4}$ that can be implemented by Pauli gates, the Hadamard gate, a CNOT gate and general single-qubit three-parameter rotation gates. The two recovery operations have the form $V_{3}=U_{1}^{\dagger} H$ and $V_{4}=H U_{2}^{\dagger} X$, where

$$
\begin{aligned}
& U_{1}=\frac{1}{\sqrt{(1+t)^{2}+(1-s)^{2}}}\left(\begin{array}{cc}
-t-1 & s-1 \\
-s+1 & -t-1
\end{array}\right), \\
& U_{2}=\frac{1}{\sqrt{(1+t)^{2}+(1-s)^{2}}}\left(\begin{array}{cc}
-s+1 & t+1 \\
-t+1 & -s+1
\end{array}\right),
\end{aligned}
$$

where the parameters $s$ and $t$ are given by

$$
s=\frac{\sqrt{2}}{\sqrt{1+(1-\gamma)^{2}}} \quad \text { and } \quad t=\frac{\sqrt{2}(1-\gamma)}{\sqrt{1+(1-\gamma)^{2}}} .
$$

The general setup of the circuit for both standard correction and optimal recovery is shown in Fig. 1. Information on the specific circuits is given in Table 2 and Fig. 3.

1. Shor, P. W. Algorithms for quantum computation: Discrete logarithms and factoring. In Proceedings 35th annual Symposium on Foundations of Computer Science, 124-134 (IEEE, 1994).

2. Lloyd, S., Mohseni, M. \& Rebentrost, P. Quantum principal component analysis. Nature Physics 10, 631 (2014).

3. Ladd, T. D. et al. Quantum computers. Nature 464, 45 (2010). 
4. Arute, F. et al. Quantum supremacy using a programmable superconducting processor. Nature 574, 505-510 (2019).

5. Wright, K. et al. Benchmarking an 11-qubit quantum computer. Nature Communications 10, 5464 (2019).

6. Preskill, J. Quantum computing in the NISQ era and beyond. arXiv preprint arXiv:1801.00862 (2018).

7. Levitt, M. H. Composite pulses. Progress in Nuclear Magnetic Resonance Spectroscopy 18, 61-122 (1986).

8. Bendall, M. R. \& Gordon, R. E. Depth and refocusing pulses designed for multipulse NMR with surface coils. Journal of Magnetic Resonance (1969) 53, 365-385 (1983).

9. Rowland, B. \& Jones, J. A. Implementing quantum logic gates with gradient ascent pulse engineering: principles and practicalities. Philosophical Transactions of the Royal Society A: Mathematical, Physical and Engineering Sciences 370, 4636-4650 (2012).

10. Calderbank, A. R. \& Shor, P. W. Good quantum error-correcting codes exist. Physical Review A 54, 1098 (1996).

11. Gottesman, D. An introduction to quantum error correction. In Proceedings of Symposia in Applied Mathematics, vol. 58, 221-236 (2002).

12. Nielsen, M. A. \& Chuang, I. L. Quantum Computation and Quantum Information: 10th Anniversary Edition (Cambridge University Press, New York, NY, USA, 2011), 10th edn.

13. Leung, D. W., Nielsen, M. A., Chuang, I. L. \& Yamamoto, Y. Approximate quantum error correction can lead to better codes. Physical Review A 56, 2567 (1997).

14. Chao, R. \& Reichardt, B. W. Quantum error correction with only two extra qubits. Physical Review Letters 121, 050502 (2018).

15. Grassl, M., Kong, L., Wei, Z., Yin, Z.-Q. \& Zeng, B. Quantum error-correcting codes for qudit amplitude damping. IEEE Transactions on Information Theory 64, 4674-4685 (2018).

16. Beale, S. J., Wallman, J. J., Gutiérrez, M., Brown, K. R. \& Laflamme, R. Quantum error correction decoheres noise. Physical Review Letters 121, 190501 (2018).

17. DiVincenzo, D. P. \& Shor, P. W. Fault-tolerant error correction with efficient quantum codes. Physical Review Letters 77, 3260 (1996).

18. Lidar, D. A., Bacon, D. \& Whaley, K. B. Concatenating decoherence-free subspaces with quantum error correcting codes. Phys. Rev. Lett. 82, 4556-4559 (1999).

19. DiVincenzo, D. P. The physical implementation of quantum computation. Fortschritte der Physik: Progress of Physics 48, 771-783 (2000). 
20. Steane, A. M. Overhead and noise threshold of fault-tolerant quantum error correction. Physical Review A 68, 042322 (2003).

21. Wang, D. S., Fowler, A. G. \& Hollenberg, L. C. Surface code quantum computing with error rates over 1\%. Physical Review A 83, 020302 (2011).

22. Barends, R. et al. Logic gates at the surface code threshold: Superconducting qubits poised for fault-tolerant quantum computing. arXiv preprint arXiv:1402.4848 (2014).

23. Braunstein, S. L. Quantum error correction for communication with linear optics. Nature 394, 47 (1998).

24. Zhang, S. et al. Error-mitigated quantum gates exceeding physical fidelities in a trapped-ion system. arXiv preprint arXiv:1905.10135 (2019).

25. Schindler, P. et al. Experimental repetitive quantum error correction. Science 332, 1059-1061 (2011).

26. Cory, D. G. et al. Experimental quantum error correction. Physical Review Letters 81, 2152 (1998).

27. Ofek, N. et al. Extending the lifetime of a quantum bit with error correction in superconducting circuits. Nature 536, 441 (2016).

28. Reed, M. D. et al. Realization of three-qubit quantum error correction with superconducting circuits. Nature 482, 382 (2012).

29. Rosenblum, S. et al. Fault-tolerant detection of a quantum error. Science 361, 266-270 (2018).

30. $\mathrm{Hu}$, L. et al. Quantum error correction and universal gate set operation on a binomial bosonic logical qubit. Nature Physics 15, 503 (2019).

31. Cardona, G., Sarlette, A. \& Rouchon, P. Continuous-time quantum error correction with noise-assisted quantum feedback. arXiv preprint arXiv:1902.00115 (2019).

32. Grassl, M., Ji, Z., Wei, Z. \& Zeng, B. Quantum-capacity-approaching codes for the detectedjump channel. Physical Review A 82, 062324 (2010).

33. Alber, G. et al. Stabilizing distinguishable qubits against spontaneous decay by detected-jump correcting quantum codes. Physical Review Letters 86, 4402 (2001).

34. Alber, G. et al. Detected-jump-error-correcting quantum codes, quantum error designs, and quantum computation. Physical Review A 68, 012316 (2003).

35. Fisher, K. A., Prevedel, R., Kaltenbaek, R. \& Resch, K. J. Optimal linear optical implementation of a single-qubit damping channel. New Journal of Physics 14, 033016 (2012). 
36. Zhao, Y.-Y., Kurzyński, P., Xiang, G.-Y., Li, C.-F. \& Guo, G.-C. Heisenberg's errordisturbance relations: A joint measurement-based experimental test. Physical Review A 95, 040101 (2017).

37. Zhao, Y.-Y. et al. Experimental detection of entanglement polytopes via local filters. $n p j$ Quantum Information 3, 11 (2017).

38. Kiesel, N., Schmid, C., Weber, U., Ursin, R. \& Weinfurter, H. Linear optics controlled-phase gate made simple. Physical Review Letters 95, 210505 (2005).

39. Okamoto, R., Hofmann, H. F., Takeuchi, S. \& Sasaki, K. Demonstration of an optical quantum controlled-NOT gate without path interference. Physical Review Letters 95, 210506 (2005).

40. Wu, K.-D. et al. Experimental cyclic interconversion between coherence and quantum correlations. Physical Review Letters 121, 050401 (2018).

41. Ježek, M., Fiurášek, J. \& Hradil, Z. Quantum inference of states and processes. Physical Review A 68, 012305 (2003).

42. Harper, R. \& Flammia, S. T. Fault-tolerant logical gates in the IBM Quantum Experience. Phys. Rev. Lett. 122, 080504 (2019).

43. Alber, G. et al. Stabilizing distinguishable qubits against spontaneous decay by detected-jump correcting quantum codes. Phys. Rev. Lett. 86, 4402-4405 (2001).

44. Verstraete, F., Dehaene, J. \& DeMoor, B. Local filtering operations on two qubits. Physical Review A 64, 010101 (2001).

Acknowledgements Q.H. Guo and Y.-Y. Zhao contributed equally to this work. We thank Dawei Lu, Shuming Cheng, Kevin Resch, Runyao Duan for fruitful discussions. Y.-Y. Zhao is supported by the National Natural Science Foundation for the Youth of China (Grants No.11804410). M. Grassl acknowledges partial support by the Foundation for Polish Science (IRAP project, ICTQT, contract no. 2018/MAB/5, co-financed by EU within the Smart Growth Operational Programme). G.-Y. Xiang is supported by the National Natural Science Foundation of China (Grants No.11574291, 11774334). T. Xin is supported by National Natural Science Foundation of China (Grants No. 11905099 and No. U1801661), and Guangdong Basic and Applied Basic Research Foundation (Grant No. 2019A1515011383). Z.-Q. Yin is supported by National Natural Science Foundation of China (Grants No. 61771278) and Beijing Institute of Technology Research Fund Program for Young Scholars.

We gratefully acknowledge use of the IBM Q for this work. The views expressed are those of the authors and do not reflect the official policy or position of IBM or the IBM Q team.

Competing Interests The authors declare that they have no competing financial interests.

Correspondence Correspondence and requests for materials should be addressed to G.-Y.X. (email: gyxiang@ustc.edu.cn), T.X. (email: xint@ sustech.edu.cn) or B.Z. (email: zengb@ust.hk). 


\section{Supplemental Material: Testing a Quantum Error-Correcting Code on Various Platforms}

\section{The Amplitude Damping Channel}

The amplitude damping channel is an important model that describes spontaneous emission and the loss of energy in quantum communication $\underline{S 12 \mid S 43}$. Generally, the effect of s channel on a quantum state is represented by a completely positive, trace-preserving linear map acting on a density matrix $\rho$. Such a map can be expressed in the Kraus representation $\mathcal{A}(\rho)=\sum_{i} A_{i} \rho A_{i}^{\dagger}$ with $\sum_{i} A_{i}^{\dagger} A_{i}=I$. The Kraus representation of the single-qubit amplitude damping channel has the form

$$
\rho \rightarrow \rho^{\prime}=\mathcal{A}_{A D}(\rho)=A_{0} \rho A_{0}^{\dagger}+A_{1} \rho A_{1}^{\dagger},
$$

where the Kraus operators are

$$
A_{0}=\left(\begin{array}{cc}
1 & 0 \\
0 & \sqrt{1-\gamma}
\end{array}\right) \text { and } A_{1}=\left(\begin{array}{cc}
0 & \sqrt{\gamma} \\
0 & 0
\end{array}\right)
$$

The operation $A_{1}$ maps the state $|1\rangle$ to the state $|0\rangle$, corresponding to the entire loss of energy of a qubit, e.g., due the the spontaneous emission of a photon. The operation $A_{0}$ does not change the state $|0\rangle$, but reduces the amplitude of the state $|1\rangle$. For the detected amplitude damping channel, the additional classical information is available which of the two cases has occurred. To simulate a detected amplitude damping channel, we have to apply the operation $A_{0}$ or $A_{1}$ to the state, which are are non-unitary local operation. hence we need local filters ${ }^{\mathrm{S} 44}$ in quantum optics or add ancillas for the superconducting circuit and NMR system ${ }^{[\mathrm{S12}}$, making use of post selection.

\section{Post Selection and State Tomography}

For each qubit, there are the two possible errors $A_{0}$ and $A_{1}$, which in our simulation of the channel can be distinguished by the outcome of measuring the ancillas. In total, four different error patterns $A_{i} A_{j}=A_{i} \otimes A_{j}$ may occur on the encoded bipartite state. To be more concrete, assume that the initial state $|\psi\rangle$ was prepared on qubit $A$. Then the encoding operation gives the encoded two-qubit state $\rho_{A, B}$ on qubits $A$ and $B$. Suppose that the channels act on both qubits of the encoded state $\rho_{A, B}$. Then we will obtain the density matrix $\rho_{i j}^{\prime}$ with probability $p_{i j}$, where

$$
\begin{aligned}
& \rho_{i j}^{\prime}=\frac{A_{i} A_{j} \rho_{A, B} A_{i}^{\dagger} A_{j}^{\dagger}}{\operatorname{Tr}\left(A_{i} A_{j} \rho_{A, B} A_{i}^{\dagger} A_{j}^{\dagger}\right)}, \\
& p_{i j}=\operatorname{Tr}\left(A_{i} A_{j} \rho_{A, B} A_{i}^{\dagger} A_{j}^{\dagger}\right),
\end{aligned}
$$

for $i, j=0,1$. To recover the information of the quantum state, we decode the density matrix $\rho_{i j}^{\prime}$ and then trace out qubit $B$. The final reduced density matrix $\rho_{A}^{\prime}$ can be obtained by taking 
the weighted sum of the results of single qubit state tomography on the qubit $A$ for the four cases $i, j=0,1$. Therefore, the final density matrix $\rho_{A}^{\prime}$ has the form

$$
\rho_{A}^{\prime}=\sum_{i, j} p_{i j} \operatorname{Tr}_{B}\left[\mathcal{D}\left(\frac{A_{i} A_{j} \rho_{A, B} A_{i}^{\dagger} A_{j}^{\dagger}}{\operatorname{Tr}\left(A_{i} A_{j} \rho_{A, B} A_{i}^{\dagger} A_{j}^{\dagger}\right)}\right)\right],
$$

where $\mathcal{D}$ denotes the decoding operation. To measure the distance between the final density matrix $\rho_{A}^{\prime}$ and the initial quantum state $\rho_{A}=|\psi\rangle\langle\psi|$, we use the fidelity $F\left(\rho_{A}, \rho_{A}^{\prime}\right)$, defined as

$$
F\left(\rho, \rho_{A}^{\prime}\right)=\operatorname{Tr}\left(\rho_{A}^{1 / 2} \rho_{A}^{\prime} \rho_{A}^{1 / 2}\right)=\left\langle\psi\left|\rho_{A}^{\prime}\right| \psi\right\rangle
$$

In order to compute the average fidelity of the whole communication system, it suffices to consider the six input states

$$
\begin{aligned}
|H\rangle & =|0\rangle, & |V\rangle & =|1\rangle \\
|D\rangle & =\frac{1}{\sqrt{2}}(|0\rangle+|1\rangle), & |A\rangle & =\frac{1}{\sqrt{2}}(|0\rangle-|1\rangle) \\
|R\rangle & =\frac{1}{\sqrt{2}}(|0\rangle+i|1\rangle), & |L\rangle & =\frac{1}{\sqrt{2}}(|0\rangle-i|1\rangle)
\end{aligned}
$$

which are the states of three mutually unbiased bases forming a 2-design.

\section{Optimal Recovery}

We encode the state $|0\rangle$ as $|++\rangle$ and the state $|1\rangle$ as $|--\rangle$. Then the encoding isometry is given by $\rho \mapsto E \rho E^{\dagger}$, where

$$
E=|++\rangle\langle 0|+|--\rangle\langle 1|
$$

Combining encoding and the amplitude damping channel, we obtain Kraus operators

$$
t_{i j}=\left(A_{i} \otimes A_{j}\right) E
$$

where $i, j=0,1$.

Writing $t_{i j}$ in its polar decomposition

$$
t_{i j}=v_{i j}\left|t_{i j}\right|
$$

where $\left|t_{i j}\right|=\sqrt{t_{i j}^{\dagger} t_{i j}}$ and $v_{i j}$ are isometries, the recovery operations are given by

$$
R_{i j}(\rho)=v_{i j}^{\dagger} \rho v_{i j}+\rho_{i j} \operatorname{tr}\left(\rho\left(I-v_{i j} v_{i j}^{\dagger}\right)\right)
$$

where $\rho_{i j}$ are arbitrary single qubit states. 
As $\rho_{i j} \operatorname{tr}\left(\rho\left(I-v_{i j} v_{i j}^{\dagger}\right)\right)=0$ for all $\rho=t_{i j} \rho_{s} t_{i j}^{\dagger}$ (where $\rho_{s}$ is an arbitrary single qubit state), we will only need to implement $v_{i j}^{\dagger}$, together with an arbitrary completion to a POVM. The isometries $v_{i j}$ are given by

$$
\begin{gathered}
v_{00}=\frac{1}{2}\left(\begin{array}{cc}
\frac{\sqrt{2}}{\sqrt{1+(1-\gamma)^{2}}} & \frac{\sqrt{2}}{\sqrt{1+(1-\gamma)^{2}}} \\
1 & -1 \\
1 & -1 \\
\frac{\sqrt{2}(1-\gamma)}{\sqrt{1+(1-\gamma)^{2}}} & \frac{\sqrt{2}(1-\gamma)}{\sqrt{1+(1-\gamma)^{2}}}
\end{array}\right), v_{01}=\frac{1}{\sqrt{2}}\left(\begin{array}{cc}
1 & -1 \\
0 & 0 \\
1 & 1 \\
0 & 0
\end{array}\right) \\
v_{10}=\frac{1}{\sqrt{2}}\left(\begin{array}{cc}
1 & -1 \\
1 & 1 \\
0 & 0 \\
0 & 0
\end{array}\right), v_{11}=\frac{1}{\sqrt{2}}\left(\begin{array}{cc}
1 & 1 \\
1 & -1 \\
0 & 0 \\
0 & 0
\end{array}\right)
\end{gathered}
$$

It can be shown that $v_{00}^{\dagger}$ cannot be directly implemented in a unitary way, i.e., by a two-qubit unitary followed by tracing out one qubit, using a single CNOT operation.

We finally obtain the single-qubit gates $V_{i}$ for the error correction circuit shown in Fig. 3 ,

$$
\begin{gathered}
V_{1}=\frac{1}{\sqrt{2}}\left(\begin{array}{cc}
1 & -1 \\
1 & 1
\end{array}\right), V_{2}=\frac{1}{\sqrt{2}}\left(\begin{array}{cc}
1 & 1 \\
-1 & 1
\end{array}\right), \\
V_{3}=U_{1}^{\dagger} H, V_{4}=H U_{2}^{\dagger} X,
\end{gathered}
$$

where

$$
\begin{aligned}
& U_{1}=\frac{1}{\sqrt{(1+t)^{2}+(1-s)^{2}}}\left(\begin{array}{cc}
-t-1 & s-1 \\
-s+1 & -t-1
\end{array}\right), \\
& U_{2}=\frac{1}{\sqrt{(1+t)^{2}+(1-s)^{2}}}\left(\begin{array}{cc}
-s+1 & t+1 \\
-t+1 & -s+1
\end{array}\right),
\end{aligned}
$$

where the parameters $s$ and $t$ are given by

$$
s=\frac{\sqrt{2}}{\sqrt{1+(1-\gamma)^{2}}}, t=\frac{\sqrt{2}(1-\gamma)}{\sqrt{1+(1-\gamma)^{2}}} .
$$

In order to implement the decoding isometry

$$
D=E^{\dagger}=|0\rangle\langle++|+| 1\rangle\langle--|,
$$

one can measure the second qubit in the computational basis, followed by a Hadamard transformation $H$ on the first qubit. If $|0\rangle$ is obtained in the measurement, then the state of the first qubit is $\alpha^{\prime}|0\rangle+\beta^{\prime}|1\rangle$. If $|1\rangle$ is obtained, we have $\alpha^{\prime}|0\rangle-\beta^{\prime}|1\rangle$, so a $Z$-gate has to be applied before the single-qubit state tomography. 


\section{Preparation of the Encoded States for the Optical Platform}

In Table 1, we list the optical elements needed to prepare the six different encoded states from a polarization entangled two-phton state.

\section{Recovery Operations}

In Table 2 we list the local operations $V_{i}$ for the recovery operation (see Fig. 3). For Optimal Recovery, only the operations for the case $A_{0} A_{0}$ are different. 


\begin{tabular}{|c|c|c|}
\hline Initial state & Optical elements (qubit 1) & Optical elements (qubit 2) \\
\hline$\frac{1}{\sqrt{2}}(|0\rangle+|1\rangle)$ & HWP@ @45 & None \\
\hline$\frac{1}{\sqrt{2}}(|0\rangle-|1\rangle)$ & None & None \\
\hline$|0\rangle$ & PBS, HWP@22.5 & PBS, HWP@22.5 \\
\hline$|1\rangle$ & PBS, HWP@-22.5 & PBS, HWP@-22.5 \\
\hline$\frac{1}{\sqrt{2}}(|0\rangle+i|1\rangle)$ & QWP@0 $0^{\circ}, \mathrm{HWP} @ 22.5^{\circ}$ & HWP@22.5 \\
\hline$\frac{1}{\sqrt{2}}(|0\rangle-i|1\rangle)$ & 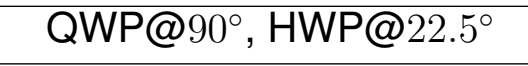 & HWP@22.5 \\
\hline
\end{tabular}

Table 1: Optical elements needed to prepare the various encoded states from a polarizationentangled two-photon state.

\begin{tabular}{|c|l|l|l|l|l|l|}
\hline Correction Type & Error Pattern & $V_{1}$ & $V_{2}$ & $V_{3}$ & $V_{4}$ & $P$ \\
\hline Standard Correction & $A_{0} A_{1}$ & $I$ & $I$ & $H X$ & $I$ & $I$ \\
\hline & $A_{1} A_{0}$ & $I$ & $I$ & $H X$ & $H$ & $X$ \\
\hline (A) & $A_{1} A_{1}$ & $I$ & $H$ & $I$ & $I$ & $I$ \\
\hline (B) & $A_{1} A_{1}$ & $I$ & $I$ & $H$ & $I$ & $I$ \\
\hline & $A_{0} A_{0}$ & $I$ & $I$ & $H$ & $H$ & $I$ \\
\hline Optimal Recovery & $A_{0} A_{0}$ & $I$ & $H$ & $U_{1}^{\dagger} H$ & $H U_{2}^{\dagger} X$ & $Z$ \\
\hline
\end{tabular}

Table 2: Recovery operations for the general setup. The table lists the specific setup for the correction and decoding circuit for the corresponding error patterns. 


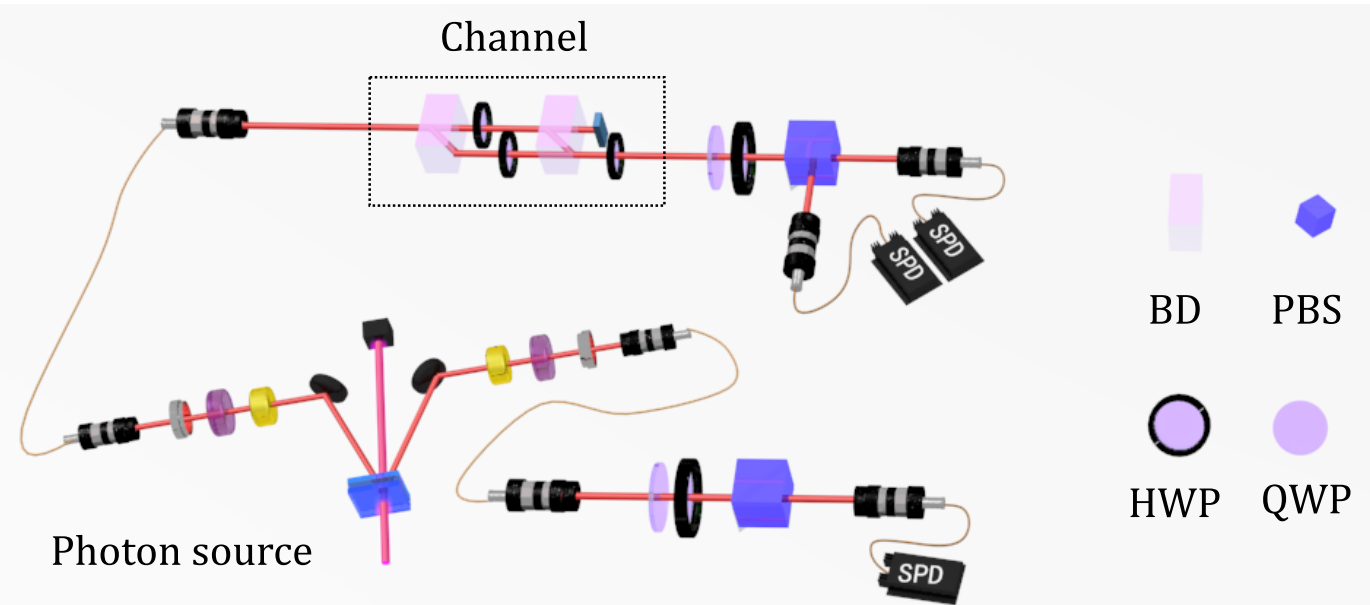

Figure S1: Experimental setup for the process without correction (optical platform)

\section{Additional Information on the optical platform, IBM Q, and the NMR System}

Detected-jump channel without correction The experimental setup for the case without correction is given in Fig. S1. The entangled photon state $1 / \sqrt{2}(|H H\rangle+|V V\rangle)$ is prepared through spontaneous parametric down-conversion (SPDC) which is similar to the photon source in Fig. 2 (a) in the main text. Then one photon of each photon pair is sent to the detected amplitude channel with the other one providing a trigger signal. Here we test the six single qubit states $|0\rangle$, $|1\rangle, 1 / \sqrt{2}(|0\rangle+|1\rangle), 1 / \sqrt{2}(|0\rangle-|1\rangle), 1 / \sqrt{2}(|0\rangle+i|1\rangle)$ and $1 / \sqrt{2}(|0\rangle-i|1\rangle)$, which can be prepared by projecting the trigger photon onto $|H\rangle,|V\rangle, 1 / \sqrt{2}(|H\rangle+|V\rangle), 1 / \sqrt{2}(|H\rangle-|V\rangle)$, $1 / \sqrt{2}(|H\rangle+i|V\rangle)$, and $1 / \sqrt{2}(|H\rangle-i|V\rangle)$, respectively. At last, standard single qubit tomography is performed on the photon passing through the channel.

Error probabilities for IBM Q In Table 3, we list the error probabilities for IBM Q.

Information about NMR system In Table 4, we list the frequencies (Hz) and coupling constants for the Crotonic acid used in the NMR experiment. 


\begin{tabular}{|l|l|l|l|l|}
\hline Error Type & $Q_{5}$ & $Q_{6}$ & $Q_{8}$ & $Q_{9}$ \\
\hline single-qubit gate error $\left(10^{-3}\right)$ & 2.36 & 1.73 & 1.81 & 3.44 \\
\hline readout error $\left(10^{-2}\right)$ & 4.30 & 3.88 & 3.14 & 7.72 \\
\hline & CX5_6 & CX6_8 & CX5_9 & CX9_8 \\
\hline multi-qubit gate error $\left(10^{-2}\right)$ & 6.90 & 5.63 & 6.49 & 5.36 \\
\hline
\end{tabular}

Table 3: Error probabilities for IBM Q 16 Melbourne: gate-error and and readout-error information for qubits $Q_{5}, Q_{6}, Q_{8}, Q_{9}$, as well as the error rates for the control gates CX5_6, CX5_9, CX6_8 and CX9_8, as provided on 2018-12.

\begin{tabular}{|l|l|l|l|l|}
\hline & $C_{1}$ & $C_{2}$ & $C_{3}$ & $C_{4}$ \\
\hline$C_{1}$ & 2560.603 & & & \\
\hline$C_{2}$ & 41.65 & 21837.66 & & \\
\hline$C_{3}$ & 1.47 & 69.73 & 18494.94 & \\
\hline$C_{4}$ & 7.03 & 1.17 & 72.35 & 25144.73 \\
\hline
\end{tabular}

Table 4: Chemical shifts (Hz) of the $i$ th spin and the $J$-coupling strength between spins $i$ and $j$ of the Crotonic acid used in the NMR experiment. 


\section{Estimate of Decoherence Errors}

For a two-qubit gate, depolarization due to thermal noise can be estimated from the relaxation times $T_{1}$ and $T_{2}$ for each qubit. For the state $|++\rangle$, a simple model shows that the density matrix evolves as

$$
\rho(t)=\frac{1}{4}\left(\begin{array}{cccc}
1 & 0 & 0 & e^{-2 t / T_{2}} \\
0 & 1 & e^{-2 t / T_{2}} & 0 \\
0 & e^{-2 t / T_{2}} & 1 & 0 \\
e^{-2 t / T_{2}} & 0 & 0 & 1
\end{array}\right) .
$$

The infidelity of the first qubit is

$$
P_{\text {sys }}\left(t, T_{2}\right)=\frac{1}{2}-\frac{1}{2} e^{-2 t / T_{2}}
$$

For IBM Q 16 Melbourne, the average time for a CNOT gate is approximately $348 \mathrm{~ns}$, while the time for a single qubit rotation is about $100 \mathrm{~ns}$. A buffering time of $20 \mathrm{~ns}$ has to be added before and after each gate. So the total time on IBM Q is nearly $2680 \mathrm{~ns}$. The decoherence error estimate for IBM Q is about $3.5 \%$. For the NMR system, we use GRAPE to generate a pulse with duration time $61 \mathrm{~ms}$, with an error estimate of $9.9 \%$. 


\section{Detailed Results}

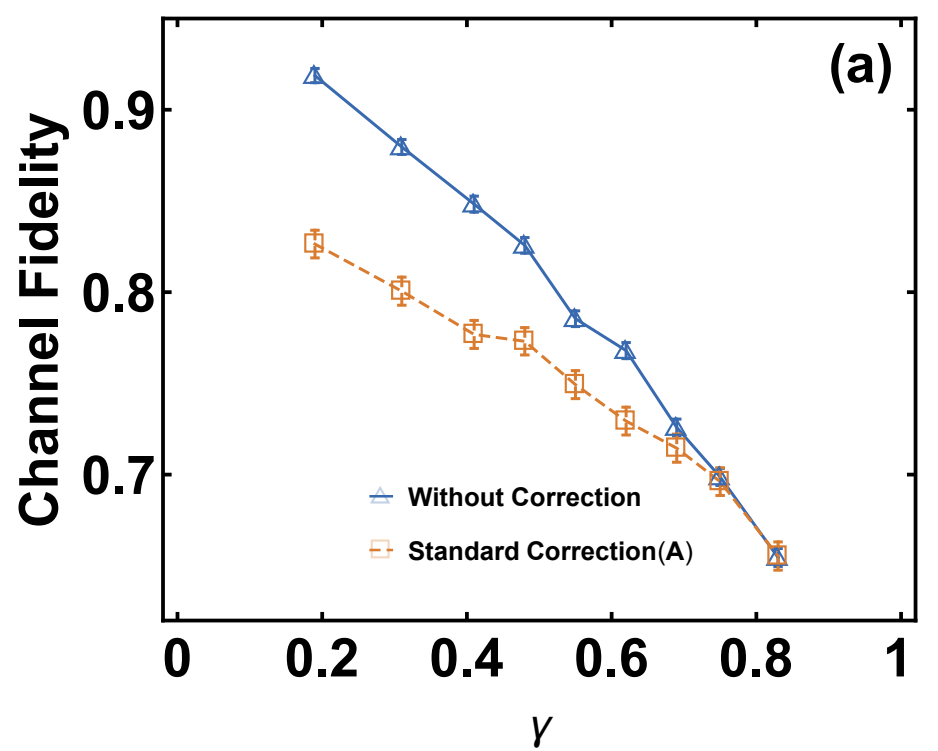

(a) Channel fidelities

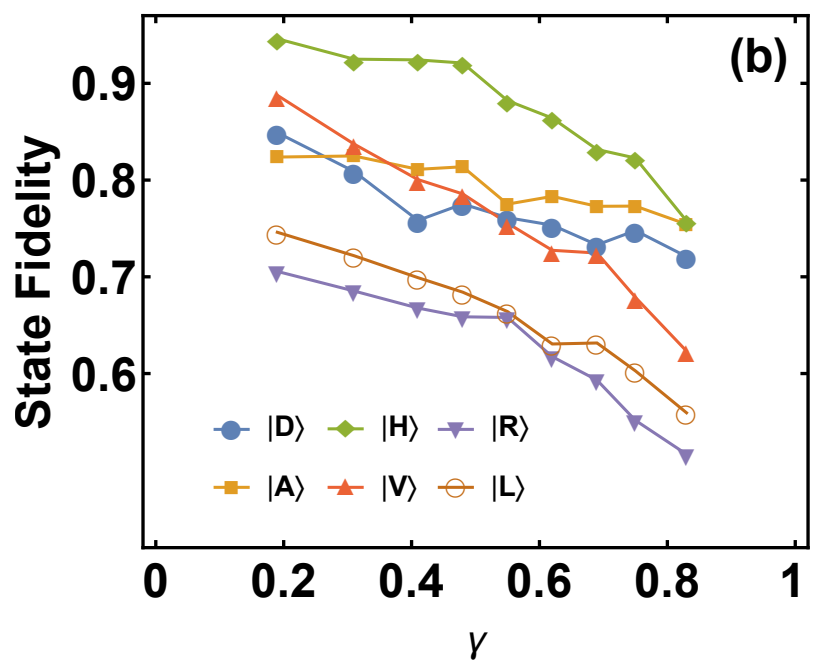

(b) Standard Correction

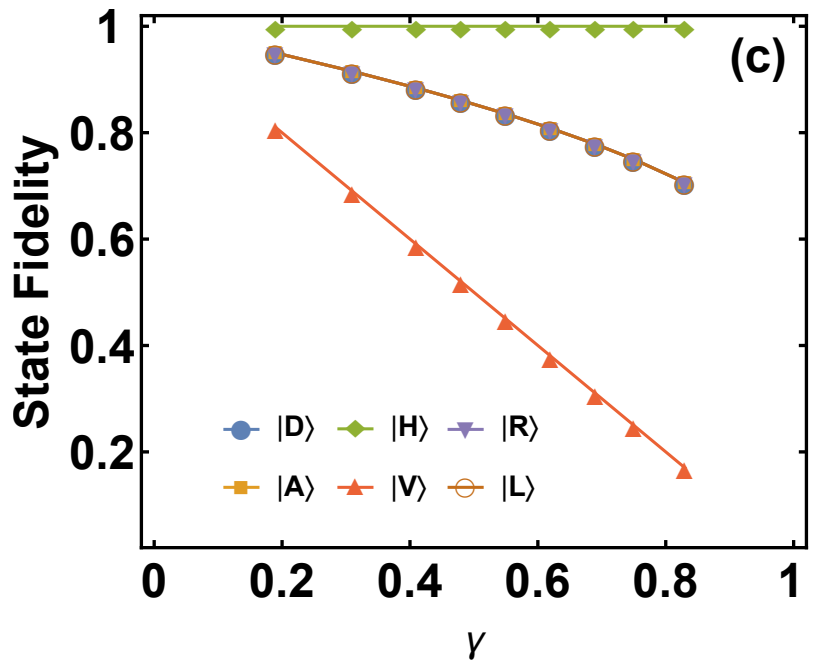

(c) Without Correction

Figure S2: Experimental results from the optical platform. Standard Correction takes effect until $\gamma$ reaches 0.83 . Error bars are obtained by Monte Carlo simulation (10000 shots). 


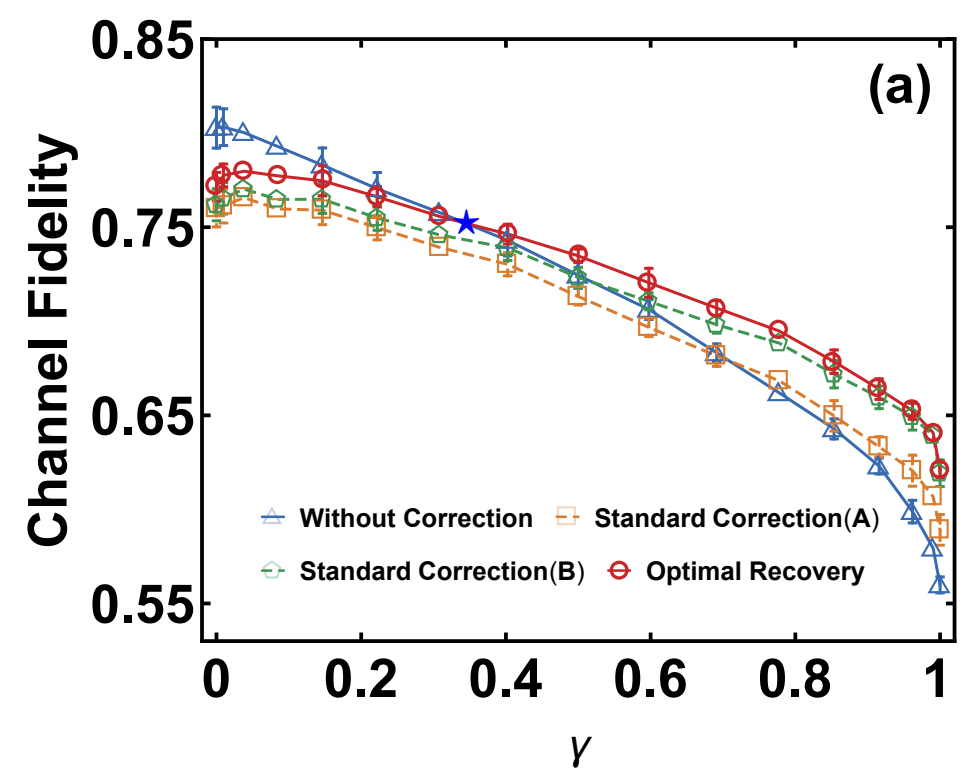

(a) Channel fidelities

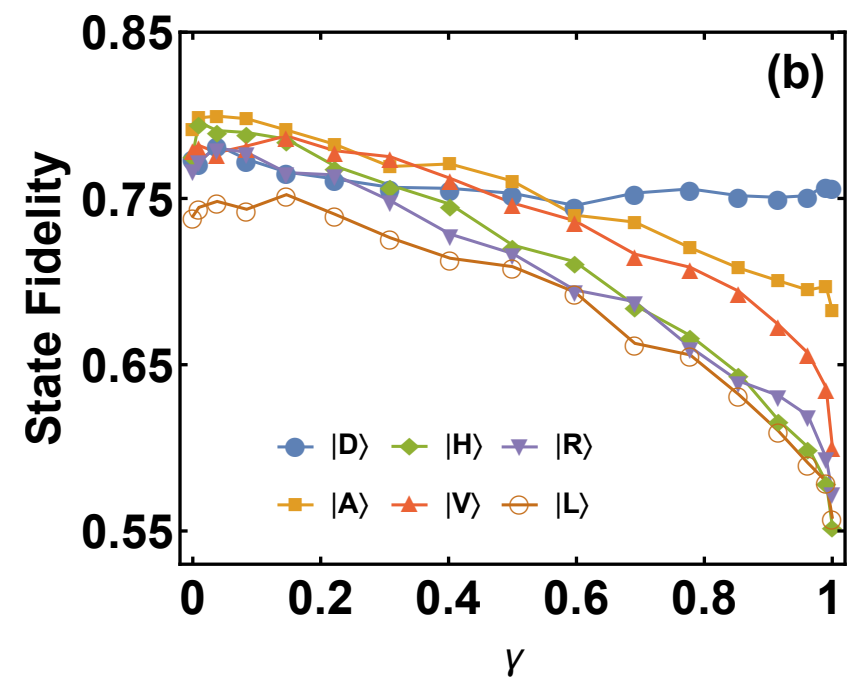

(b) Optimal Recovery

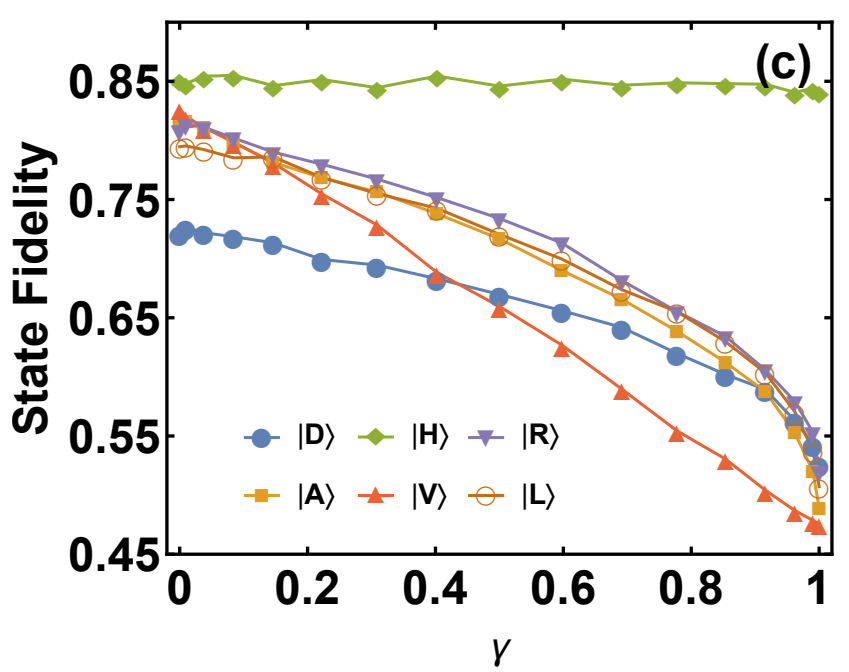

(c) Without error correction

Figure S3: Experimental results from IBM Q. Optimal Recovery shows a dominance at large damping ratio $\gamma$. The error bars in Fig. 4 (a) are derived from the standard deviation via bootstrapping. 


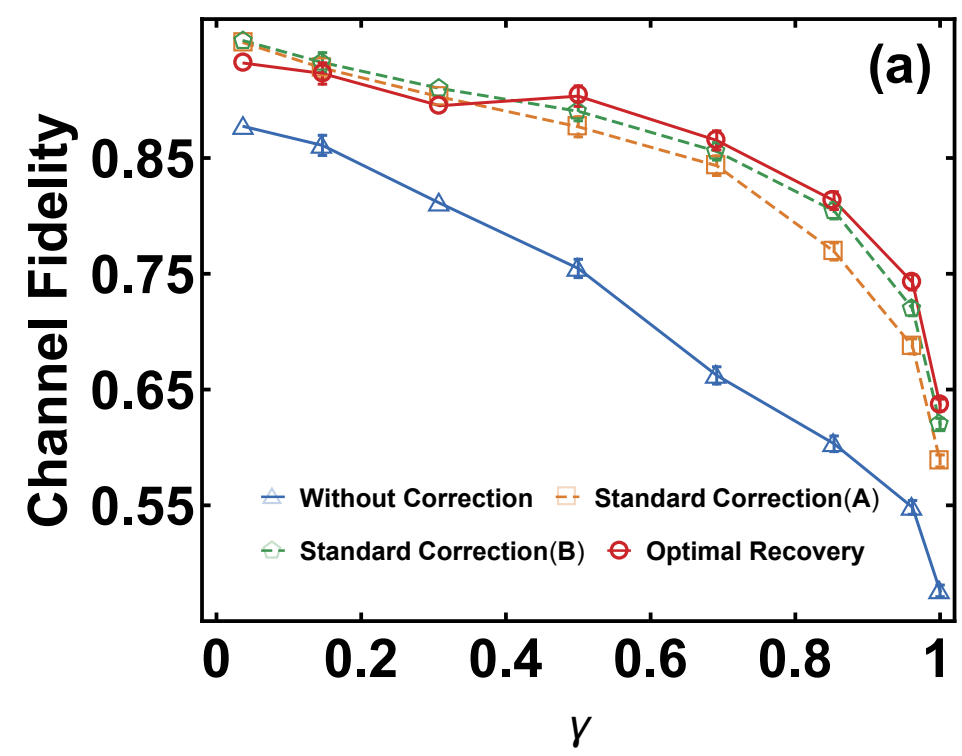

(a) Channel fidelities

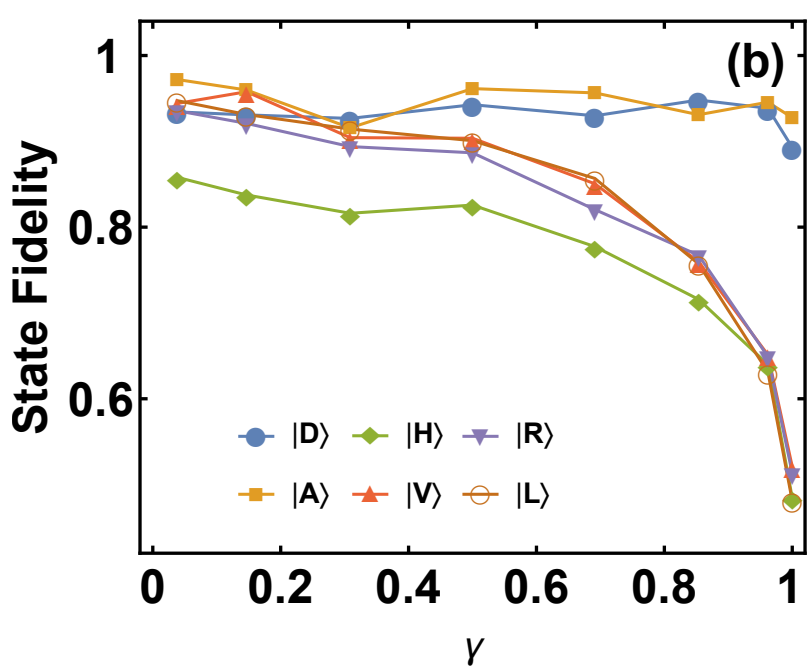

(b) Optimal Recovery

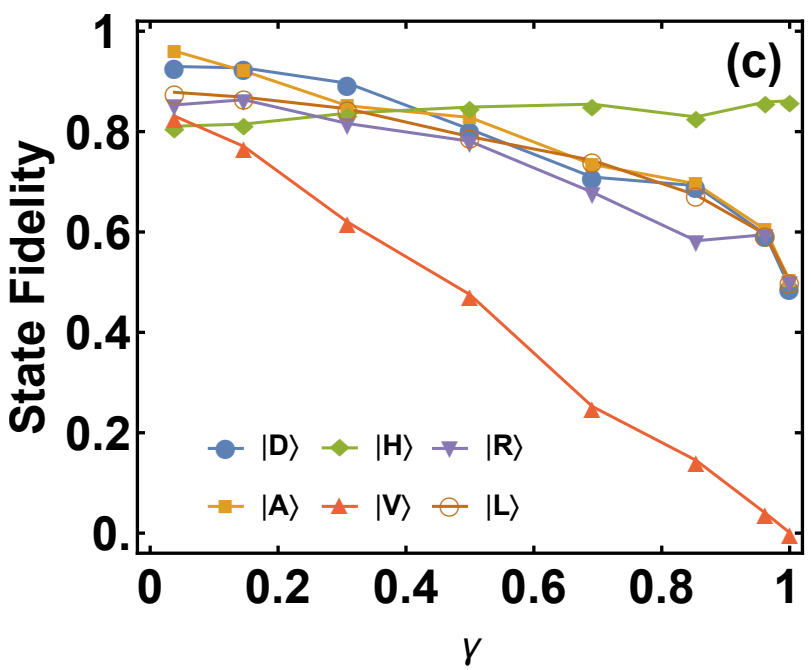

(c) Without Correction

Figure S4: Experimental results from the NMR system. The experiment on the NMR system verifies the power of Optimal Recovery. The error bars in diagram (a) are from random samples. 


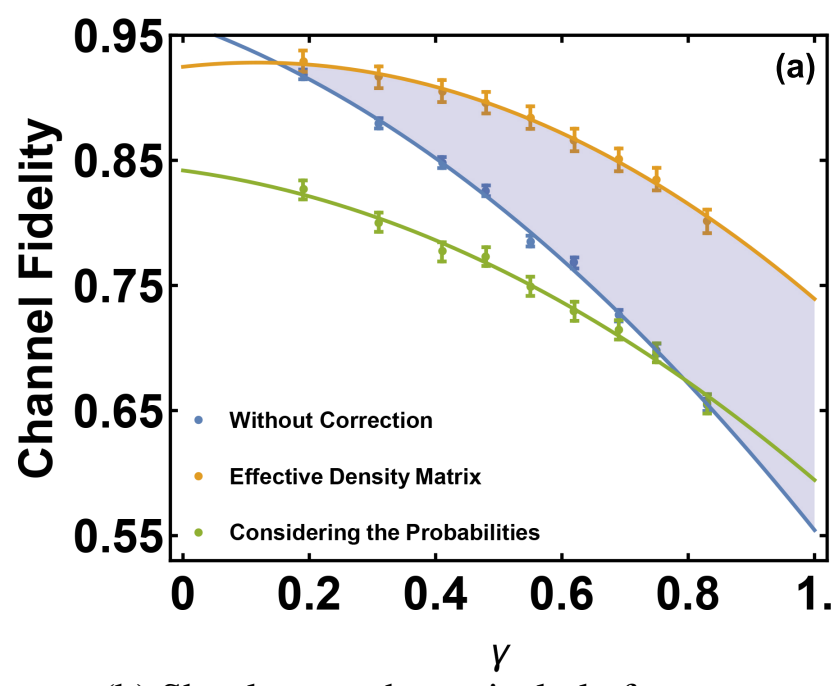

(b) Shot loss on the optical platform

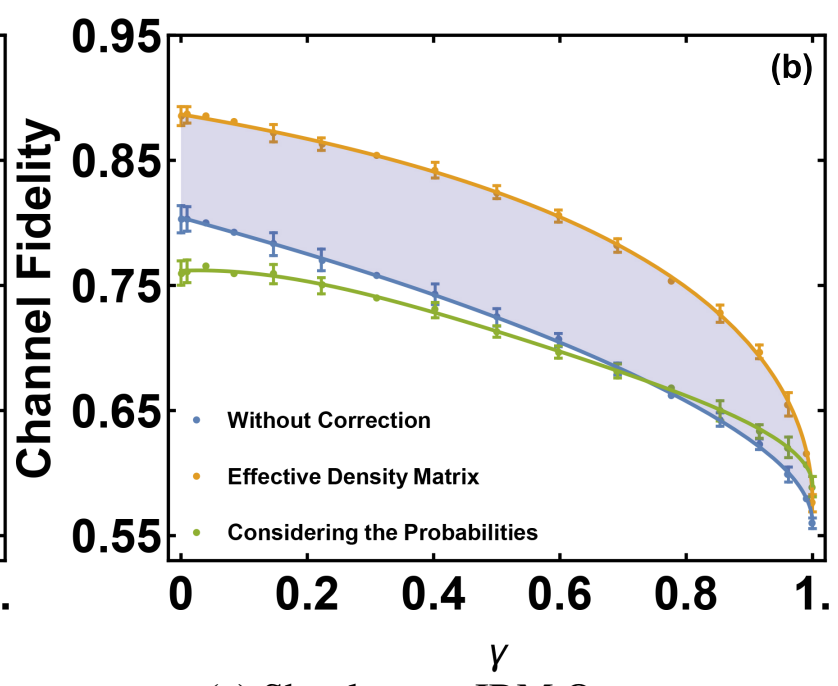

(a) Shot loss on IBM Q

Figure S5: Shot loss of CNOT. (a) On the optical platform, the partially polarizing beam splitters (PPBS) mainly contribute to the loss of photons. Compared with the raw single-qubit density matrix, the fidelity of the density matrix incorporating the loss declines a lot. (b) On IBM Q, the loss of shots results from the crosstalk between qubits, and leads to some abnormal excitation on other qubits. We compare the fidelity of the raw single-qubit density matrix with that of the real density matrix considering the probabilities. 